\title{
THE GEOMETRIC DIMENSION OF SOME VECTOR BUNDLES OVER PROJECTIVE SPACES $\left({ }^{1}\right)$
}

\author{
BY \\ DONALD M. DAVIS AND MARK E. MAHOWALD
}

ABSTRACT. We prove that in many cases the geometric dimension of the p-fold Whitney sum $\mathrm{pH}_{k}$ of the Hopf bundle $H_{k}$ over quaternionic projective space $Q P^{k}$ is the smallest $n$ such that for all $i \leqslant k$ the reduction of the $i$ th symplectic Pontryagin class of $p H_{k}$ to coefficients $\pi_{4 i-1}\left(\left(R P^{\infty} / R P^{n-1}\right) \wedge b o\right)$ is zero, where bo is the spectrum for connective $K O$-theory localized at 2 . We immediately obtain new immersions of real projective space $R P^{4 k+3}$ in Euclidean space if the number of 1 's in the binary expansion of $k$ is between 5 and 8 .

1. Introduction. In this paper we determine the geometric dimension (gd) of the stable class of many of the multiples $p H_{k}$ of the Hopf bundle $H_{k}$ over quaternionic projective space $Q P^{k}$. This enables us immediately to obtain some new immersions of real projective spaces $R P^{4 k+3}$ in Euclidean space.

Let $\nu\left(2^{a}(2 b+1)\right)=a, \nu(p, i)=\nu\left({ }_{i}^{p}\right)$, and let $\alpha(n)$ equal the number of 1 's in the binary expansion of $n$. Let $P_{n}^{m}=\mathbf{R} P^{m} / R P^{n-1}$ and $P_{n}=P_{n}^{\infty}$. bo denotes the spectrum for real connective $K$-theory localized at $2[3] . \pi_{4 i-1}\left(P_{n} \wedge b o\right)$ is a finite cyclic group ([j] or [7]).

THEOREM 1.1. (a) If $\operatorname{gd}\left(p H_{k}\right) \leqslant n$, then $\nu(p, i) \geqslant \nu\left(\pi_{4 i-1}\left(P_{n} \wedge b o\right)\right)$ for all $i \leqslant k$.

(b) The converse of (a) is true if $n \geqslant 2 k$ and $\pi_{4 i-1}^{s}\left(P_{n}\right) \rightarrow \pi_{4 i-1}\left(P_{n} \wedge\right.$ bo $)$ is injective for all $i \leqslant k$.

REMARK 1.2. $\operatorname{gd}\left(p H_{k}\right) \geqslant 2 k$, because if the stable class of $p H_{k}$ contains a $2 k$-plane bundle, it has nonzero Euler class, since $e^{2}=p_{k} \neq 0$.

By using the computations of [7] or [3] and [11], the observation that $\left(\begin{array}{l}p \\ i\end{array}\right)$ is the coefficient of the symplectic Pontryagin class $e_{i}\left(p H_{k}\right)$, and an argument which is presented in $\S 4$ to eliminate one extraneous obstruction, Theorem 1.1 may be restated:

Presented to the Society, January 18, 1974; received by the editors February 8, 1974.

AMS (MOS) subject classifications (1970). Primary 55G35, 55G40, 55G45, 57A35; Secondary 55G25.

Key words and phrases. Geometric dimension, immersions of projective spaces, symplectic Pontryagin classes, connective $K$-theory, modified Postnikov towers.

(1) This research was supported by NSF grant GP 25335 .

Copyright $\odot$ 1975. American Mathemattcal Siciety 
THEOREM 1.3. (a) If $\operatorname{gd}\left(p H_{k}\right) \leqslant 4 m+\epsilon(\epsilon=1,2,3)$, then for all $m+j$ $\leqslant k, e_{m+j}\left(p H_{k}\right)$ is divisible by $2^{2 j}$ if $j$ even, $2^{2 j+2-\epsilon}$ if $j$ odd.

(b) The converse is true if $4 m+\epsilon \geqslant 2 k$ and

$$
m \geqslant k- \begin{cases}3 & \text { if } \epsilon=1, \text { and } \nu(p, m) \geqslant 3 \text { if } m \text { even, } \\ 4 \quad \text { if } \epsilon=2,3, \text { and } \nu(p, m+1) \geqslant 3 \text { if } m \text { odd } .\end{cases}
$$

This enables us to state the precise geometric dimension of many $p H_{k}$, for example $\operatorname{gd}\left(16 \mathrm{H}_{4}\right)=\operatorname{gd}\left(16 \mathrm{H}_{5}\right)=14$ and $\operatorname{gd}\left(18 \mathrm{H}_{10}\right)=\operatorname{gd}\left(18 \mathrm{H}_{11}\right)=39$. Part (a) is slightly stronger than the main theorem of [14]. We conjecture that part (b) is true without the condition $m \geqslant k-\left\{\begin{array}{l}3 \\ 4\end{array}\right.$.

We obtain new immersions of $R P^{4 k+3}$ by combining Theorem 1.3(b) with the map $R P^{4 k+3} \rightarrow Q P^{k}$.

THEOREM 1.4. If $K \equiv 3(4)$, then $R P^{K} \subseteq \mathrm{R}^{2 K-D}$, where $D$ is given by the following table:

\begin{tabular}{l|rrrrr}
$\alpha(K)$ & 6 & 7 & 8 & 9 & 10 \\
\hline$D$ & 10 & 12 & 13 & 14 & 17
\end{tabular}

These results for $\alpha(K) \geqslant 7$ are slightly stronger than those of Milgram [12]. The first new immersion result that we obtain is $\mathbf{R} P^{191} \subseteq \mathbf{R}^{370}$. These results are not quite as good as the conjectured best possible immersion dimension:

CONJECTURE 1.5. [7]. If $K \equiv 7$ (8) the smallest Euclidean space in which $R P^{K}$ can be immersed as dimension

$$
2 K-2 \alpha(K)+\left\{\begin{aligned}
0 & \text { if } \alpha(K) \equiv 0(4) \\
1 & \text { if } \alpha(K) \equiv 1,2(4) \\
-1 & \text { if } \alpha(K) \equiv 3(4)
\end{aligned}\right.
$$

We feel that the conjectured immersions for $\alpha(K) \leqslant 10$ will follow quite readily from Theorem 1.3(b) together with computations of the indeterminacies in the modified Postnikov tower [6]. As these computations are extremely detailed, they will be deferred until a later paper. The negative part of this conjecture has been announced and retracted [4], [7]. It is hoped that the methods of this paper together with those of the last sections of [5] will produce a proof of the negative part of this conjecture.

The rationale behind this conjecture is the belief that only bo-primary homotopy of $V_{n}$ should obstruct multiples of the line bundle over projective spaces and that these obstructions should be given in terms of the symplectic Pontryagin classes. Some attempts have been made to show the latter directly using $K$-theoretic $e_{i}$-classes. In this paper we have shown that as far as divisibility by 2 is concerned, the $e_{i}$ could be the obstructions. 
The argument can be sketched as follows.

THEOREM 1.6. There are bundles

$$
V_{n} \wedge \text { bo } \rightarrow E_{n}^{o} \rightarrow B S p, \quad V_{n} \wedge b u \rightarrow E_{n}^{u} \rightarrow B S p
$$

such that

(i) there are pairings of bundles

$$
E_{n}^{o} \times E_{m}^{o} \rightarrow E_{n+m}^{o}, \quad E_{n}^{u} \times E_{m}^{u} \rightarrow E_{n+m}^{u} ;
$$

(ii) if $Q P^{k} \stackrel{f}{\longrightarrow}$ BSp classifies a stable real vector bundle $\theta$, then $\operatorname{gd}(\theta) \leqslant n$ implies $f$ lifts to $E_{n}^{o}$, and if $f$ lifts to $E_{n}^{o}$ and $n \geqslant 2 k$ and $\pi_{4 i-1}\left(P_{n}\right) \rightarrow$ $\pi_{4 i-1}\left(P_{n} \wedge\right.$ bo) is injective for all $i \leqslant k$, then $\operatorname{gd}(\theta) \leqslant n$.

DEFINITION 1.7. $N(p, k)$ is the smallest $N$ such that

$$
\nu(p, i) \geqslant \nu\left(\pi_{4 i-1}\left(P_{N} \wedge b o\right)\right) \text { for all } i \leqslant k \text {. }
$$

$M(p, k)$ is the smallest $M$ such that $\nu(p, i) \geqslant \nu\left(\pi_{4 i-1}\left(P_{M} \wedge b u\right)\right)$ for all $i \leqslant k$.

Theorem 1.1 then follows from Theorem 1.6 and

THEOREM 1.8. $p H_{k}$ lifts to $E_{\max (2 k, N(p, k))}^{o}$ but not to $E_{\max }^{o}(2 k, N(p, k))-1$ -

This is proved inductively by noting that if $p=\Sigma_{i=1}^{r} 2^{e_{i}}$, with all $e_{i}$ distinct if $p$ is not a power of 2 , and $e_{1}=e_{2}=i$ if $p=2^{i+1}$, then $p H_{k}$ is classified by the composite

$$
\begin{aligned}
Q P^{k} & \stackrel{\Delta}{\longrightarrow}\left(Q P^{k} \times \cdots \times Q P^{k}\right)^{(4 k)} \\
& =\bigcup Q P^{k_{1}} \times \cdots \times Q P^{k_{r}} \stackrel{\times 2^{e_{i} H}}{\longrightarrow} B S p \times \cdots \times B S p \rightarrow B S p .
\end{aligned}
$$

where $\Delta$ is a skeletal map homotopic to the iterated diagonal, and the union is taken over all ordered $r$-tuples $\left\langle k_{1}, \ldots, k_{r}\right\rangle$ such that $\Sigma k_{i}=k$. Liftings of $X 2^{e_{i}} H_{k_{i}}$ to $E_{M(p, k)}^{u}$ are found by using the liftings of the factors and the pairing of Theorem 1.6(i). These fit together to give a lifting of $p H_{k}$ to $E_{M(p, k)}^{u}$. The results for lifting to $E_{N}^{o}$ are obtained by using the maps $E_{N}^{o} \rightarrow E_{N}^{u}$.

2. Construction of some spaces and maps. Let $\widetilde{B S} p_{n}$ be the fibered product defined by the diagram

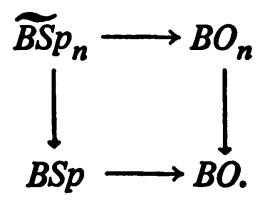


We wish to form the fiberwise smash product [10] of $\widetilde{B S} p_{n}$ with the bo-spectrum. In order to form the fiberwise smash, the original bundle must have a section, which we obtain by applying fiberwise unreduced suspension, but then we must be careful in order to have a fiberwise map from the original bundle into the new one.

Let $b_{l}$ indicate the $l$ th space in a connective ringed $\Omega$-spectrum $b$ [17]. Then there is a map $S^{l} \rightarrow b_{l}$, the unit of the ring, which commutes with $\epsilon: b_{l} \rightarrow \Omega b_{l+1}$ and with the pairing $\mu: b_{l_{1}} \wedge b_{l_{2}} \rightarrow b_{l_{1}+l_{2}}$. Let $x_{l} \in \Omega^{l} b_{l}$ correspond to this map. Let $F \rightarrow E \stackrel{D}{\longrightarrow} B$ be a fiber bundle. Form

$$
\Omega_{B}^{l}\left(\left(S_{B}^{n} E\right) \wedge_{B} b_{l}\right) \stackrel{\bar{p}_{n}}{\longrightarrow} B
$$

as in [10]. Let $P_{B}^{n} \Omega_{B}^{l}\left(\left(S_{B}^{n} E\right) \wedge_{B} b_{l}\right)$ be the space of maps

$$
\sigma: I^{n} \rightarrow \Omega_{B}^{l}\left(\left(S_{B}^{n} E\right) \wedge_{B} b_{l}\right)
$$

such that $\bar{p}_{n} \sigma$ is constant and for $\bar{s} \in I^{n}, \bar{t} \in I^{l}, \sigma(\mathcal{S})(\bar{t})=\left[\bar{s}, p^{-1} \bar{p}_{n}\left(\sigma\left(I^{n}\right)\right), x_{l}(\bar{t})\right]$. Then there is a fiberwise map $i_{E}: E \rightarrow P_{B}^{n} \Omega_{B}^{l}\left(\left(S_{B}^{n} E\right) \wedge_{B} b_{l}\right)$ given by $i_{E}(e)(\bar{s}, \bar{t})=$ $\left[\bar{s}, e, x_{2}(\bar{t})\right]$. Also, there are obvious maps

$$
P_{B}^{n} \Omega_{B}^{l}\left(\left(S_{B}^{n} E\right) \wedge_{B} b_{l}\right) \rightarrow P_{B}^{n} \Omega_{B}^{l+1}\left(\left(S_{B}^{n} E\right) \wedge_{B} b_{l+1}\right)
$$

so that we can form

$$
\left(E \wedge_{B} b\right)_{n}=\lim _{l} P_{B}^{n} \Omega_{B}^{l}\left(\left(S_{B}^{n} E\right) \wedge_{B} b_{l}\right)
$$

Note that

$$
\text { fiber } \begin{aligned}
\left(\left(E \wedge \wedge_{B} b\right)_{n} \rightarrow B\right) & =\lim P^{n} \Omega^{l}\left(S^{n} F \wedge b_{l}\right) \simeq \lim \Omega^{l+n}\left(\Sigma^{n} F \wedge b_{l}\right) \\
& \simeq \lim _{l} \Omega^{l+n}\left(F \wedge b_{l+n}\right)=F \wedge b,
\end{aligned}
$$

since $\Sigma^{n} b_{l} \rightarrow b_{l+n}$ is a $(2 l-n)$-equivalence. (Here $P^{n} \Omega^{l}\left(S^{n} F \wedge b_{l}\right)$ is the set of maps $\sigma: I^{n} \times I^{l} \rightarrow S^{n} F \wedge b_{l}$ such that $\bar{s} \in I^{n}$ implies $\sigma(\bar{s}, \bar{t})=\left[\bar{s}, F, x_{l}(\bar{t})\right]$ and $\bar{t} \in I^{l}$ implies $\sigma(\bar{s}, \bar{t})=*$. The equivalence with $\Omega^{n+l}\left(\Sigma^{n} F \wedge b_{l}\right)$ is obtained by reducing the suspensions.)

Given a pairing of fiber bundles

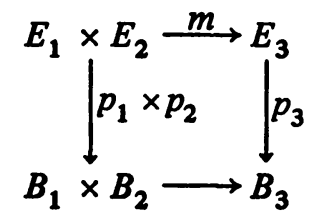


we define maps

$$
\begin{gathered}
\bar{m}:\left(\left(S_{B_{1}}^{n_{1}} E_{1}\right) \wedge_{B_{1}} b_{l_{1}}\right) \times\left(\left(S_{B_{2}}^{n_{2}} E_{2}\right) \wedge_{B_{2}} b_{l_{2}}\right) \rightarrow\left(\left(S_{B_{3}}^{n_{1}+n_{2}} E_{3}\right) \wedge_{B_{3}} b_{l_{1}+l_{2}}\right), \\
P_{B_{1}}^{n_{1}} \Omega_{B_{1}}^{l_{1}}\left(\left(S_{B_{1}}^{n_{1}} E_{1}\right) \wedge_{B_{1}} b_{l_{1}}\right) \times P_{B_{2}}^{n_{2}} \Omega_{B_{2}}^{l_{2}}\left(\left(S_{B_{2}}^{n_{2}} E_{2}\right) \wedge_{B_{2}} b_{l_{2}}\right) \\
\mid \bar{m} \\
P_{B_{3}}^{n_{1}+n_{2}} \Omega_{B_{3}}^{l_{1}+l_{2}}\left(\left(S_{B_{3}}^{n_{1}+n_{2}} E_{3}\right) \wedge_{B_{3}} b_{l_{1}+l_{2}}\right)
\end{gathered}
$$

by

$$
\begin{gathered}
\bar{m}\left(\left[\bar{s}_{1}, e_{1}, y_{1}\right],\left[\bar{s}_{2}, e_{2}, y_{2}\right]\right)=\left[\left(\bar{s}_{1}, \bar{s}_{2}\right), m\left(e_{1}, e_{2}\right), \mu\left(y_{1}, y_{2}\right)\right], \\
\overline{\bar{m}}\left(\sigma_{1}, \sigma_{2}\right)\left(\bar{s}_{1}, \bar{s}_{2}, \bar{t}_{1}, \bar{t}_{2}\right)=\bar{m}\left(\sigma_{1}\left(\bar{s}_{1}, \bar{t}_{1}\right), \sigma_{2}\left(\bar{s}_{2}, \bar{t}_{2}\right)\right) .
\end{gathered}
$$

Since $P_{B}^{n} \Omega_{B}^{l}\left(\left(S_{B}^{n} E\right) \wedge_{B} b_{l}\right) \rightarrow\left(E \wedge_{B} b\right)_{n}$ is an $(l-n)$ equivalence, by choosing $l_{1}$ and $l_{2}$ large, $\bar{m}$ may be regarded as defining a bundle pairing

$$
\left(E_{1} \wedge_{B_{1}} b\right)_{n_{1}} \times\left(E_{2} \wedge_{B_{2}} b\right)_{n_{2}} \rightarrow\left(E_{3} \wedge_{B_{3}} b\right)_{n_{1}+n_{2}}
$$

through any finite skeleta, which is compatible with $m$, since $i_{E_{3}} m=\bar{m}\left(i_{E_{1}} \times i_{E_{2}}\right)$. Let

$$
E_{n}^{o}=\left(\widetilde{B S p_{n}} \wedge_{B S p} b o\right)_{n} \text { and } E_{n}^{u}=\left(\widetilde{B S p_{n}} \wedge_{B S p} b u\right)_{n}
$$

Thus, there are commutative diagrams of fiber bundles:
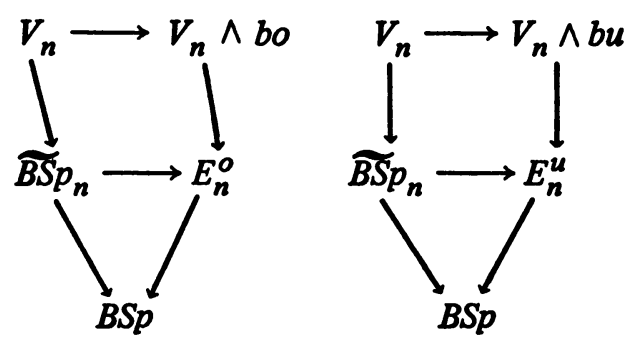

where $V_{n}=\lim _{k} V_{n+k, k}$. The pairings of Theorem 1.6(i) now follow by the construction of the previous paragraph, since the Whitney sum pairing $B O_{n} \times B O_{m} \rightarrow$ $B O_{n+m}$ induces a map $\widetilde{B S p}_{n} \times \widetilde{B S p}_{m} \rightarrow \widetilde{B S p_{n+m}}$. Theorem 1.6(ii) follows easily since $\operatorname{gd}(\theta) \leqslant n$ if and only if $f$ lifts to $\widehat{B S p_{n}}$. There is a well-known map [9] $P_{n} \rightarrow V_{n}$ which is a $2 n$ equivalence. Thus in the stable range the only 
obstructions to lifting $f$ from $E_{n}^{o}$ to $\widetilde{B S}_{n}$ are due to the non-bo-primary homotopy of $P_{n}$ in dimensions $4 i-1$. Note that for $n$ odd all the homotopy of $P_{n}$ is'2-primary, while for $n$ even and $p$ an odd prime, the $p$-primary homotopy of $P_{n}$ cannot cause an obstruction by naturality from the lifting problem for $E_{n-1}^{o}$.

THEOREM 2.1. For $\Delta \leqslant 4$ there are equivalences of skeleta

$$
\begin{aligned}
& \left(E_{n}^{o} / E_{n-\Delta}^{o}\right)^{(2(n-\Delta)+1)} \rightarrow \underset{I}{\bigvee}\left(\Sigma^{4|I|+1} P_{n-\Delta}^{n-1} \wedge b o\right)^{(2(n-\Delta)+1)}, \\
& \left(E_{n}^{u} / E_{n-\Delta}^{u}\right)^{(2(n-\Delta)+1)} \rightarrow \underset{I}{\bigvee}\left(\Sigma^{4|I|+1} P_{n-\Delta}^{n-1} \wedge b u\right)^{(2(n-\Delta)+1)}
\end{aligned}
$$

where I ranges over $\{0\}$ and all sets of positive integers and $|I|$ is the sum of all elements of $I$.

REMARK. This theorem seems to be true for arbitary $\Delta$ but we shall just need it for $\Delta \leqslant 4$.

Proof. As noted by Moore [13], there is a spectral sequence converging to $H^{*}\left(\widetilde{B S p_{n}}, \widetilde{B S p_{n-\Delta}} ; \mathrm{Z}_{2}\right)$ with $E_{2}^{p, q}=H^{p}\left(B S p ; H^{q}\left(V_{n}, V_{n-\Delta}\right)\right)$, which in the stable range is $H^{p}\left(B S p ; H^{q}\left(\Sigma P_{n-\Delta}^{n-1}\right)\right)$. The map $\left(\widetilde{B S p_{n}}, \widetilde{B S} p_{n-\Delta}\right) \stackrel{f}{\rightarrow}$ $\left(B O_{n}, B O_{n-\Delta}\right)$ induces a map of spectral sequences. Since there are no differentials in the spectral sequence of $\left(B O_{n}, B O_{n-\Delta}\right)$ in the stable range, there are none in that of $\left(\widetilde{B S} p_{n}, \widetilde{B S} p_{n-\Delta}\right)$. Moreover, it follows from naturality of the external cup product that in the stable range $H^{*}\left(\widetilde{B S} p_{n}, \widetilde{B S p_{n-\Delta}}\right) \approx H^{*}(B S p) \otimes H^{*}\left(\Sigma P_{n-\Delta}^{n-1}\right)$ as modules over the subalgebra $A_{1}$ of the mod 2 Steenrod algebra generated by $\mathrm{Sq}^{1}$ and $\mathrm{Sq}^{2}$. Since $\Delta \leqslant 4$ each $H^{q}\left(\widetilde{B S p_{n}}, \widetilde{B S p_{n-\Delta}}\right), q \leqslant 2(n-\Delta)+1$, contains elements of only one filtration, so there is no ambiguity in writing an element as an element of $H^{*}(B S p) \otimes H^{*}\left(\Sigma P_{n-\Delta}^{n-1}\right)$. Then, for example, in $H^{*}\left(\widetilde{B S p}_{4 m+5}, \widetilde{B S p_{4 m+1}}\right)$,

$$
\begin{aligned}
\mathrm{Sq}^{2}\left(e_{1} \otimes s a_{4 m+3}\right) & =\mathrm{Sq}^{2}\left(f^{*}\left(w_{4} \cup w_{4 m+4}\right)\right)=f^{*}\left(\left(w_{6}+w_{2} w_{4}\right) \cup w_{4 m+4}\right) \\
& =\bar{f}^{*}\left(w_{6}+w_{2} w_{4}\right) \cup f^{*}\left(w_{4 m+4}\right)=0,
\end{aligned}
$$

where $\bar{f}: \widetilde{B S p_{n}} \rightarrow B O_{n}$.

The $E_{2}$-term of the Adams spectral sequence for $\pi_{*}\left(\left(\widetilde{B S} p_{n} / \widetilde{B S} p_{n-\Delta}\right) \wedge b o\right)$ is

$$
\operatorname{Ext}_{A_{1}}^{s, t}\left(H^{*}\left(\widetilde{B S} p_{n}, \widetilde{B S} p_{n-\Delta}\right), Z_{2}\right) \approx \operatorname{Ext}_{A_{1}}^{s, t}\left(H^{*}\left(V \Sigma^{4|I|+1} P_{n-\Delta}^{n-1}\right), Z_{2}\right) \text {. }
$$

These groups can be read off from [3]. There can be no differentials by naturality, since there are no possible differentials in the Adams spectral sequence for $\widetilde{B S p_{n-\epsilon+1}} / \widetilde{B S p_{n-\epsilon}} \wedge b o, 1 \leqslant \epsilon \leqslant \Delta$. 
We can use our determination of $\pi_{*}\left(\widetilde{B S p_{n}} / \widetilde{B S p}_{n-\Delta} \wedge b o\right)$ to map

$$
\vee \Sigma^{4|I|+1} P_{n-\Delta}^{n-1} \stackrel{\vee f_{I}}{\longrightarrow} \widetilde{B S} p_{n} / \widetilde{B S} p_{n-\Delta} \wedge \text { bo }
$$

by using the Barratt-Puppe sequence to extend the map cell-by-cell. For example, consider the case $\widetilde{B S p_{4 m+5}} / \widetilde{B S p_{4 m+1}} \cdot \pi_{q}\left(\widetilde{B S p_{4 m+s}} / \widetilde{B S p_{4 m+1}} \wedge b o\right)(q \leqslant 4 m+14)$ can be pictured as below, where dots indicate nontrivial classes and vertical lines indicate multiplication by 2 . (See [3].)

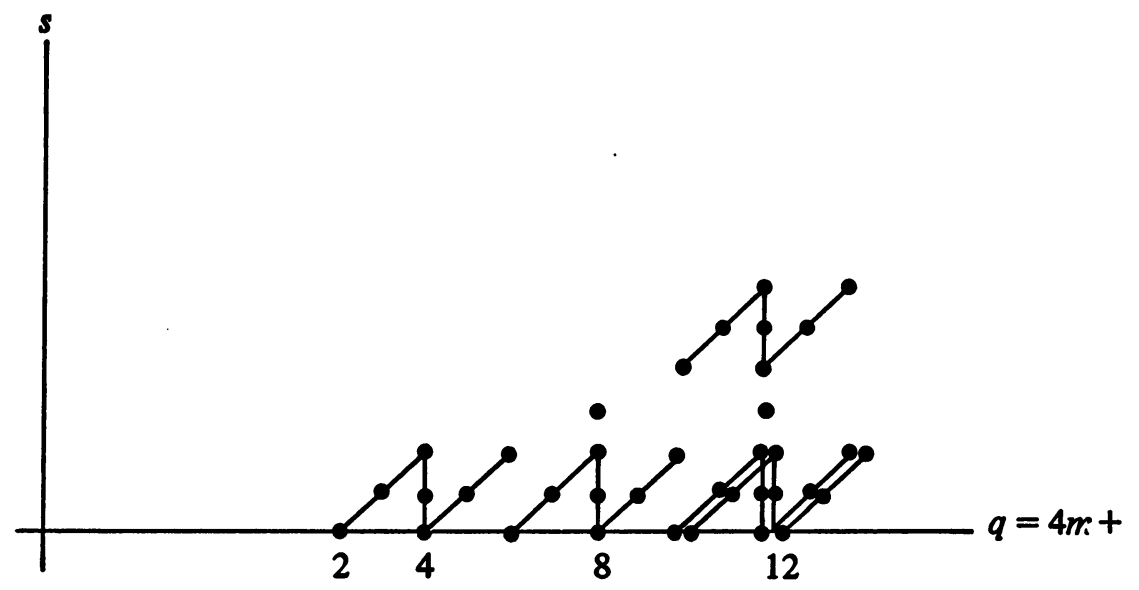

Thus for each $I$ there is a map

$$
S^{4|I|+4 m+2} \vee S^{4|I|+4 m+4} \stackrel{f_{I}}{\longrightarrow} \widetilde{B S p} p_{4 m+5} / \widetilde{B S p}_{4 m+1} \wedge \text { bo }
$$

such that $f_{I}^{*}\left(e_{I} \otimes s a_{n} \otimes 1\right)=g_{4|I|+r}$ for $r=4 m+2,4$. $2 f_{I} \mid S^{4|I|+4 m+2} \simeq 0$ so that $f_{I}$ extends over $S^{4|I|+4 m+2} \cup_{2} e^{4|I|+4 m+3} \vee S^{4|I|+4 m+4}$. The map

$$
S^{4|X|+4 m+4} \stackrel{\bar{\eta}}{\longrightarrow} S^{4|I|+4 m+2} U_{2} e^{4|I|+4 m+3}
$$

generates $\pi_{4|I|+4 m+4}\left(S^{4|I|+4 m+2} \cup_{2} e^{4|I|+4 m+3}\right) \approx \mathbf{Z}_{4} . f_{I} \bar{\eta}$ is thus twice the generator of the appropriate summand of $\pi_{4|I|+4 m+4}\left(\widetilde{B S} p_{4 m+5} / \widetilde{B S p}_{4 m+1} \wedge b o\right)$ and hence equals $2 f_{I} \mid S^{4|I|+4 m+4}$. Thus $f_{I}$ extends over

$$
S^{4|I|+4 m+2} U_{2} e^{4|I|+4 m+3} \vee S^{4|I|+4 m+4} U_{2, \bar{\eta}} e^{4|I|+4 m+5} \approx \Sigma^{4|I|+1} P_{4 m+1}^{4 m+4}
$$

The composition

$$
\begin{aligned}
\bigvee \Sigma^{4|I|+1} P_{4 m+1}^{4 m+4} \wedge \text { bo } & \stackrel{\bigvee f_{I} \wedge I}{\longrightarrow} \widetilde{B S p}_{4 m+5} / \widetilde{B S p_{4 m+1}} \wedge b o \wedge b o \\
& \stackrel{1 \wedge \mu}{\longrightarrow} \widetilde{B S p}_{4 m+5} / \widetilde{B S p_{4 m+1}} \wedge b o
\end{aligned}
$$


induces an isomorphism in $\mathbf{Z}_{\mathbf{2}}$-cohomology through the stable range, and since bo is localized at 2 , it is a $(8 m+3)$ equivalence. But

$$
E_{4 m+5}^{o} / E_{4 m+1}^{o} \approx \widetilde{B S p_{4 m+5}} / \widetilde{B S p}_{4 m+1} \wedge \text { bo. }
$$

A similar argument works when bo is replaced by $b u$.

THEOREM 2.2. (i) For $\Delta \leqslant 4$ there are maps $\left(E_{n}^{o}\right)^{(2(n-\Delta)+1)} \rightarrow \Sigma P_{n-\Delta}^{n-1} \wedge$ bo whose fiber has the same $2(n-\Delta)$-type as $E_{n-\Delta}^{o}$, and similarly for $E_{n}^{u}$.

(ii) The following diagram is homotopy commutative, where the horizontal maps are induced by complexification bo $\rightarrow$ bu.

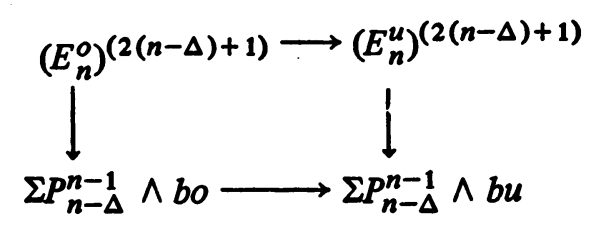

(iii) For $\Delta=1$, the maps $\left(E_{n}^{u}\right)^{2 n-1} \stackrel{h_{n}}{\longrightarrow} S^{n} \wedge$ bu can be chosen so that the diagrams

$$
\begin{aligned}
&\left(E_{2 n}^{u}\right)^{4 n-1} \times\left(E_{2 m}^{u}\right)^{4 m-1} \rightarrow\left(E_{2(n+m)}^{u}\right)^{4 n+4 m-1} \\
& h_{2 n} \times h_{2 m}\left.\right|_{2(n+m)} \\
& S^{2 n} \wedge b u \wedge S^{2 m} \wedge b u \rightarrow S^{2 n+2 m} \wedge b u
\end{aligned}
$$

are homotopy commutative.

Proof. Let $E$ be the fiber of the composite

$$
E_{n}^{o(2(n-\Delta)+1)} \rightarrow E_{n}^{o} / E_{n-\Delta}^{o(2(n-\Delta)+1)} \stackrel{h}{\longrightarrow} \vee \Sigma^{4}{ }^{(U 1+1} P_{n-\Delta}^{n-1} \rightarrow \Sigma P_{n-\Delta}^{n-1} \wedge \text { bo, }
$$

where $h$ is a homotopy inverse of the map constructed in Theorem 2.1. Through dimension $2(n-\Delta)$ there is a commutative diagram of fibrations:

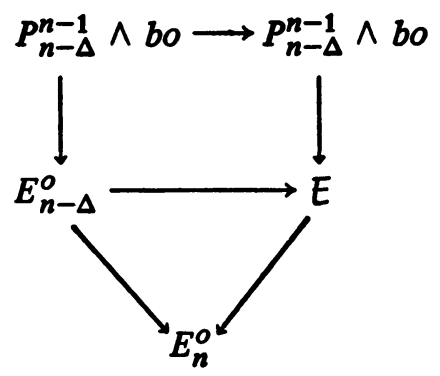


In both fibrations the fundamental class $a_{n-\Delta} \otimes 1$ transgresses nontrivially, and since it is the only nonzero cohomology class in its dimension it must be mapped to itself. But all classes in $H^{*}\left(P_{n-\Delta}^{n-1} \wedge b o ; Z_{2}\right)$ are related to one another by the action of the Steenrod algebra, and hence the map of fibers must be a $2(n-\Delta)$ equivalence. Thus $E_{n-\Delta}^{o} \rightarrow E$ is a $2(n-\Delta)$ equivalence.

Part (ii) follows by functorality of complexification; i.e., a map

$$
\widetilde{B S p_{n}} / \widetilde{B S p_{n-\Delta}} \wedge \text { bo } \rightarrow \Sigma P_{n-\Delta}^{n-1} \wedge \text { bo }
$$

induces a compatible map

$$
\widetilde{B S} p_{n} / \widetilde{B S} p_{n-\Delta} \wedge b u \rightarrow \Sigma P_{n-\Delta}^{n-1} \wedge b u,
$$

which can be thought of as smashing with the identity map of $S^{0} U_{\eta} e^{2}$.

(iii) It suffices to find elements $x_{2 n} \in K_{U}^{o}\left(\widetilde{B S}_{2 n}\right)$ satisfying

(a) the restriction to $K_{U}^{o}\left(\widetilde{B S p_{2 n-1}}\right)$ of $x_{2 n}$ is zero,

(b) $m *\left(x_{2(n+m)}\right)=x_{2 n} \otimes x_{2 m}$, where $m$ is the natural pairing $\widetilde{B S} p_{2 n} \times$ $\widetilde{B S p}_{2 m} \rightarrow \widetilde{B S p} p_{2 n+2 m}$,

(c) $x_{2 n}$ has filtration $2 n$ and is represented by a map $\widetilde{B S} p_{2 n} \rightarrow S^{2 n} \wedge b u$ which is nontrivial in $\mathbf{Z}_{\mathbf{2}}$-cohomology.

We then let $h_{n}$ be the composite

$$
E_{2 n}^{u} \rightarrow \widetilde{B S p_{2 n}} \wedge b u \stackrel{x_{2 n} \wedge 1}{\longrightarrow} S^{2 n} \wedge b u \wedge . b u \stackrel{1 \wedge \mu}{\longrightarrow} S^{2 n} \wedge b u .
$$

The elements $y_{2 n} \in K_{\cup}^{o}\left(B \operatorname{Spin}_{2 n}\right)$ corresponding to the representation $\Delta_{2 n}^{+}-$ $\Delta_{2 n}^{-}$[8] under the isomorphism $K_{\cup}^{o}\left(B \operatorname{Spin}_{2 n}\right) \approx R\left(\operatorname{Spin}_{2 n}\right)^{\wedge}$ [2] satisfy the analogues of (a), (b), (c), so we choose $x_{2 n}=g^{*} y_{2 n}$, where $g$ is the map $\widetilde{B S} p_{2 n}$ $\rightarrow B \operatorname{Spin}_{2 n}$ obtained by lifting the map $\widetilde{B S p} p_{2 n} \rightarrow B O_{2 n}$.

THEOREM 2.3. For $b=b o$ or $b u$ and any space $X$,

$$
\left[X_{i=1}^{r} Q P^{k_{i}}, X \wedge b\right] \approx \bigoplus_{l_{i}<k_{i}} \pi_{4 \Sigma l_{i}}(X \wedge b) .
$$

Proof. Let $D_{q}\left(X Q P^{k_{i}}\right)$ be a Spanier-Whitehead dual [16]. Then by [17]

$$
\left[X Q P^{k_{i}}, X \wedge b\right] \approx \pi_{q}\left(D\left(X Q P^{k_{i}}\right) \wedge X \wedge b\right) .
$$

Since $H^{q}\left(D\left(X Q P^{k} t\right) ; Z_{2}\right)$ is nonzero only every fourth dimension, as in the proof of Theorem 2.1, $D\left(X Q P^{k}\right) \wedge b \simeq \bigvee s^{q-4 \Sigma l_{i}} \wedge b$. Thus

$$
\pi_{q}\left(D\left(X Q P^{k_{i}}\right) \wedge X \wedge b\right) \approx \pi_{q}\left(\vee S^{q-4 \Sigma l_{i}} \wedge X \wedge b\right) \approx \bigoplus \pi_{4 \Sigma l_{i}}(X \wedge b) .
$$


THEOREM 2.4. There exists a generator $e_{L}(X, b)$ of the $\pi_{4 \Sigma l_{i}}(X \wedge b)$-component of $\left[X Q P^{k_{i}}, X \wedge b\right]$ for $b=b u, X=S^{2 n}, \Sigma P_{2 n-1}^{2 n}$, or $\Sigma P_{2 n-1}^{2 n+1}$ and for $b=b o, X=\Sigma P_{n}^{n+\Delta}, \Delta \leqslant 4, n+\Delta \neq 3$ (4) if $\Delta>0$ satisfying:

(i) they are natural with respect to inclusions $X Q P^{k_{i}^{\prime}} \rightarrow X Q P^{k_{i}}$;

(ii) they are natural with respect to maps $X \rightarrow X^{\prime}$ which are inclusions or collapsings and with respect to complexification bo $\rightarrow$ bu in the sense that if $\pi_{4 i}(X \wedge b) \rightarrow \pi_{4 i}\left(X^{\prime} \wedge b^{\prime}\right)$ sends generator to $2^{e}$-generator, then [ $\left[X Q P^{k_{i}}, X \wedge b\right.$ ] $\rightarrow\left[X Q P^{k_{i}}, X^{\prime} \wedge b^{\prime}\right]$ sends $e_{L}(X, b)$ to $2^{e} e_{L}\left(X^{\prime}, b^{\prime}\right)$;

(iii) if $\Delta: Q P^{k} \rightarrow\left(Q P^{k} \times \cdots \times Q P^{k}\right)^{(4 k)}$ is a skeletal map homotopic to the diagonal, then

$$
\Delta^{*}:\left[\left(Q P^{k} \times \cdots \times Q P^{k}\right)^{(4 k)}, X \wedge b u\right] \rightarrow\left[Q P^{k}, X \wedge b u\right]
$$

satisfies $\Delta^{*}\left(e_{L}\right)=e_{|L|}$, where $|L|=\Sigma l_{i}$ if $L=\left\langle l_{i}\right\rangle$;

(iv) the natural pairing $\otimes\left[Q P^{k_{i}}, S^{2 n_{i}} \wedge b u\right] \rightarrow\left[X Q P^{k_{i}}, S^{\Sigma 2 n_{i}} \wedge b u\right]$ sends $e_{l_{1}} \otimes \cdots \otimes e_{l_{r}} \mapsto e_{l_{1}}, \cdots, l_{r}$.

PROOF. As in [1, Lemma 2.5] $k_{o}^{*}\left(X_{i=1}^{r} Q P^{k}\right)$ is the truncated polynomial algebra $k_{o}^{*}(p t)\left[\alpha_{1}, \cdots, \alpha_{r}\right] / \alpha_{i}^{k_{i}+1}=0$, where $\alpha_{i} \in k^{4}\left(X Q P^{k_{i}}\right)$, and similarly for $k \dot{u}$. Thus

$$
\left[X_{i=1}^{r} Q P^{k_{i}}, S^{n} \wedge b\right]=k^{n}\left(X Q P^{k_{i}}\right) \approx \bigoplus_{l_{i}<k_{i}} \alpha^{l_{1}} \cdots \alpha^{l_{r}} \cdot \pi_{4 \Sigma l_{i}}\left(S^{n} \wedge b\right) .
$$

Let $e_{L}\left(S^{n}, b\right) \in\left[X Q P^{k} i, S^{n} \wedge b\right]$ correspond to $\alpha^{l_{1}} \cdots \alpha^{l_{r}}$ times the canonical generator of $\pi_{4 \Sigma l_{i}}\left(S^{n} \wedge b\right)$. If

$$
\pi_{4|L|}\left(S^{n} \wedge b\right) \stackrel{i_{\#}}{\longrightarrow} \pi_{4|L|}\left(\Sigma P_{n-1}^{n-1}+\Delta \wedge b\right)
$$

is surjective, let $e_{L}\left(\Sigma P_{n-1}^{n-1+\Delta}, b\right)=i_{*}\left(e_{L}\left(S^{n}, b\right)\right)$. This takes care of all $b=b u$ cases and some bo-cases. For other cases $e_{L}\left(\Sigma P_{n-1}^{n-1+\Delta}, b o\right)$ can be chosen to satisfy (ii) with respect to complexification and an appropriate collapsing map $P_{n-1}^{n-1+\Delta} \rightarrow P_{n-1+\epsilon}^{n-1+\Delta}$. Thus we have defined $e_{L}$ which satisfy (ii). They clearly satisfy (i) and (iv). (iii) is clearly true for $e_{L}\left(S^{2 n}, b u\right)$ and follows for other $e_{L}(X, b u)$ by naturality. Indeed,

$$
\begin{aligned}
\Delta^{*}\left(e_{L}(X, b u)\right) & =\Delta^{*} i_{*}\left(e_{L}(S, b u)\right)=i_{*} \Delta^{*}\left(e_{L}(S, b u)\right) \\
& =i_{*}\left(e_{|L|}(S, b u)\right)=e_{|L|}(X, b u) .
\end{aligned}
$$

DEFinition 2.5. A class $[f] \in\left[X Q P^{k_{i}}, X \wedge b\right]$ can be written uniquely as $\Sigma n_{L} e_{L}(X, b)$. We will call $n_{\left\langle k_{i}\right\rangle}$ the top component of [f], or the top obstruction if $f: X Q P^{k_{i}} \rightarrow E_{n} \rightarrow \Sigma P_{n-\Delta}^{n-1} \wedge b$. 
COROLlaRY 2.6. (i) Let $k$, $r$, and $Y=Q P^{l_{1}} \times \cdots \times Q P^{l_{s}}$ be fixed. Suppose that for all $K=\left\langle k_{i}: i=1, \ldots, r\right\rangle$ such that $\Sigma k_{i}=k$ we have compatible maps

$$
f_{K}: Q P^{k_{i}} \times \cdots \times Q P^{k_{r}} \times Y \longrightarrow X \wedge b u .
$$

These induce a map

$$
Q P^{k} \times Y \stackrel{\Delta}{\longrightarrow} Q P^{k_{1}} \times \cdots \times Q P^{k_{r}} \times Y \stackrel{f}{\longrightarrow} X \wedge b u
$$

such that the top component of $[f \Delta]$ equals the sum of the top components of the $\left[f_{K}\right]$.

(ii) If $f_{i}: Q P^{k_{i}} \rightarrow S^{2 n_{i}} \wedge$ bu and $f=X f_{i}: X Q P^{k_{i}} \rightarrow S^{2 \Sigma n_{i}} \wedge$ bu, then the top component of $[f]$ is the product of the top components of the $\left[f_{i}\right]$.

3. Proof of Theorem 1.8. It will be convenient to first prove

THEOREM 3.1. $p H_{k}$ lifts to $E_{M}^{u}$, where $M=\max (2 k, M(p, k))$ and $M(p, k)$ is defined in Definition 1.7. The class in $\bigoplus_{i=1}^{k} \pi_{4 i}\left(\sum P_{M-1}^{M} \wedge b u\right)$ corresponding to the map $Q P^{k} \stackrel{p H}{\longrightarrow} E_{M+1}^{u} \rightarrow \Sigma P_{M-1}^{M} \wedge$ bu under the isomorphism of Theorem 2.3 and Definition 2.5 has a nonzero $\pi_{4 i}$-component if and only if $\nu(p, i)<\nu\left(\pi_{4 i-1}\left(P_{M-1} \wedge b u\right)\right)$.

As outlined in the Introduction, Theorem 3.1 is proved by writing $p H_{k}$ as a Whitney sum. The combinatorial result which makes the induction work is the following theorem, whose proof is given in $\$ 4$.

THEOREM 3.2. If $e_{i}$ are distinct and $0 \leqslant k_{i} \leqslant 2^{e_{i}}$ and $\Sigma k_{i}=k$, then $\Sigma \nu\left(2^{e_{i}}, k_{i}\right) \geqslant \nu\left(\Sigma 2^{e_{i}}, k\right)$. For fixed $\left\langle e_{i}\right\rangle$ and $k$ there are an odd number of $\left\langle k_{i}\right\rangle$ as above for which equality is obtained.

Because we can only conclude the liftings of the factors in the stable range, the induction is made somewhat more complicated, requiring the following definitions.

Definition 3.3. A triple $\left(2^{e}, k_{1}, k_{2}\right)$ with $0 \leqslant k_{i} \leqslant 2^{e}$ is special if exactly one of the $k_{i}$ equals $2^{e}$ or exactly one of the $k_{i}$ satisfies, for all $l \leqslant k_{i}, 2 l+v(l)<e$.

Definition 3.4. Let $S=\left\{\left(2^{e_{i}}, k_{i}\right)\right\}$ be a finite set of ordered pairs such that $0 \leqslant k_{i} \leqslant 2^{e_{i}}$. If for two of the elements of $S$ we have $e_{\alpha}=e_{\beta}$, let

$$
S^{\prime}=\left\{\left(2^{e_{\alpha}+1}, k_{\alpha}+k_{\beta}\right)\right\} \cup\left\{\left(2^{e_{i}}, k_{i}\right) \in S: i \neq \alpha, \beta\right\},
$$

and write $S \mapsto S^{\prime}$ and let

$$
\epsilon\left(S, S^{\prime}\right)= \begin{cases}1 & \text { if }\left(2^{e} \alpha, k_{\alpha}, k_{\beta}\right) \text { is special, } \\ 0 & \text { if not. }\end{cases}
$$


There is a (not necessarily unique) sequence $\xi: S=S_{0} \mapsto S_{1} \mapsto \cdots \mapsto S_{n}$, where $S_{n}$ has distinct exponents $e_{i}$. Let

$$
\gamma^{\prime}(\xi)=\sum_{i=0}^{n-1} \epsilon\left(S_{i}, S_{i+1}\right) \text { and } \gamma(S)=\min _{\xi}\left(\gamma^{\prime}(\xi)\right) \text {, }
$$

where $\xi$ ranges over all such sequences.

LEMMA 3.5. $\nu\left(\Sigma 2^{e_{i}}, \Sigma k_{i}\right) \leqslant \Sigma \nu\left(2^{e_{i}}, k_{i}\right)+\gamma\left(\left\{\left(2^{e_{i}}, k_{i}\right)\right\}\right)$.

Proof. Let $\xi: S_{0} \mapsto \cdots \mapsto S_{n}$ with $S_{0}=\left\{\left(2^{e_{i}}, k_{i}\right)\right\}$ and $S_{n}=\left\{\left(2^{e^{\prime}}, k_{i}^{\prime}\right)\right\}$, $e_{i}^{\prime}$ distinct, be a sequence having minimal $\gamma^{\prime}$. If $S=\left\{\left(2^{e_{i}^{\prime \prime}}, k_{i}^{\prime \prime}\right)\right\}$, let $\nu(S)=$ $\Sigma v\left(2^{e_{i}^{\prime \prime}}, k_{i}^{\prime \prime}\right)$. Then

$$
\nu\left(S_{i}\right)-\nu\left(S_{i+1}\right)=\nu\left(2^{e}, k\right)+\nu\left(2^{e}, k^{\prime}\right)-\nu\left(2^{e+1}, k+k^{\prime}\right) \geqslant-\epsilon\left(S_{i}, S_{i+1}\right) .
$$

This follows immediately from the observations that $\nu\left(2^{e}, k\right)=e-\nu(k)$ if $k>0$, and $\nu\left(k+k^{\prime}\right) \geqslant \min \left(\nu(k), \nu\left(k^{\prime}\right)\right)$. Thus

$$
\begin{aligned}
\sum \nu\left(2^{e_{i}}, k_{i}\right) & =\nu\left(S_{0}\right) \geqslant \nu\left(S_{n}\right)-\sum \epsilon\left(S_{i}, S_{i+1}\right) \\
& =\sum \nu\left(2^{e_{i}^{\prime}}, k_{i}^{\prime}\right)-\gamma(S) \geqslant \nu\left(\sum 2^{e_{i}^{\prime}}, \sum k_{i}^{\prime}\right)-\gamma(S) \\
& =\nu\left(\sum 2^{e_{i}}, \sum k_{i}\right)-\gamma(S) .
\end{aligned}
$$

The last inequality is due to Theorem 3.2.

COROLLARY 3.6. $M\left(\Sigma 2^{e_{i}}, \Sigma k_{i}\right) \geqslant \Sigma M\left(2^{e_{i}}, k_{i}\right)-2 \gamma\left(\left\{\left(2^{e_{i}}, k_{i}\right)\right\}\right)$.

Proof. Since $\nu\left(\pi_{2 i-1}\left(P_{2 n} \wedge b u\right)\right)=v\left(\pi_{2 i-1}\left(P_{2 n+1} \wedge b u\right)\right)=i-n$, we have $M(p, k)=\max _{i<k, p}(4 i-2 v(p, i))$. Choose $l_{i} \leqslant k_{i}$ such that $M\left(2^{e}, k_{i}\right)=$ $4 l_{i}-2 v\left(2^{e_{i}}, l_{i}\right)$. Note $l_{l}=0$ if and only if for all $l \leqslant k_{i}, 2 l+v(l)<e$. Then

$$
\begin{aligned}
\sum M\left(2^{e_{i}}, k_{i}\right)-2 \gamma\left(\left\{\left(2^{e_{i}}, k_{i}\right)\right\}\right) & =4 \sum l_{i}-2\left(\sum \nu\left(2^{e_{i}}, l_{i}\right)+\gamma\left(\left\{\left(2^{e_{i}}, l_{i}\right)\right\}\right)\right) \\
& \leqslant 4 \sum l_{i}-2 \nu\left(\sum 2^{e_{i}}, \sum l_{i}\right) \leqslant M\left(\sum 2^{e_{i}}, \sum k_{i}\right) .
\end{aligned}
$$

Theorem 3.1 follows immediately from Theorems 3.7(b), 3.8(b) and 2.4(i).

Theorem 3.7. (a) $X{ }^{e}{ }^{e} H_{k_{i}}$ lifts to $E_{M}^{u}$, where

$$
M=\max \left(2 \sum k_{i}, M\left(\sum 2^{e_{i}}, \sum k_{i}\right)\right)+2 \gamma\left(\left\{\left(2^{e_{i}}, k_{i}\right)\right\}\right),
$$

if the product contains two or more factors.

(b) $p H_{k}$ lifts to $E_{\max (2 k, M(p, k))}^{u}$. 
Proof. It suffices to prove:

(i) If (a) is true whenever $\Sigma 2^{e_{i}} \leqslant p$ and (b) is true for $p H_{k^{\prime}}$ whenever $k^{\prime}<k$, then (b) is true.

(ii) If (b) is true whenever $p \leqslant \max \left\{2^{e_{i}}\right\}$ and (a) is true for $X 2^{e^{\prime}}{ }_{H_{k_{i}^{\prime}}}$ whenever $\Sigma 2^{e_{i}^{\prime}}=\Sigma 2^{e_{i}}$ and $\max \left\{2^{e_{i}^{\prime}}+k_{i}^{\prime}: M\left(2^{e_{i}^{\prime}}, k_{i}^{\prime}\right)<2 k_{i}^{\prime}\right\}<\max \left\{2^{e_{i}}+k_{i}: M\left(2^{e_{i}}, k_{i}\right)\right.$ $<2 k_{i}$ \}, then (a) is true.

Proof of (i) if $p$ is not a power of 2. Write $p=\Sigma_{i=1}^{r} 2^{e_{i}}$ with $e_{i}$ distinct. If $0 \leqslant k_{i} \leqslant 2^{e_{i}}$ and $\Sigma k_{i}=k$, then by hypothesis $X 2^{e_{i}} H_{k_{i}}$ lifts to $E_{\max (2 k, M(p, k))}^{u}$. Thus

$$
\bigcup_{\left\langle k_{i}\right\rangle} Q P^{k_{1}} \times \cdots \times Q P^{k_{r}} \stackrel{X_{2}{ }^{e_{i}} H}{\longrightarrow} B S p
$$

lifts to $E_{\max }^{u}(2 k, M(p, k))+1$ since the difference obstruction for matching the liftings on their intersections is an element of $H^{q}\left(X Q P ; \pi_{q}\left(P_{\max (2 k, M(p, k))+1} \wedge b u\right)\right)=0$, and hence the union lifts to $E_{\max (2 k, M(p, k))}^{u}$, since there is no obstruction for lifting a space with cohomology only in even dimensions from $E_{2 M+1}^{u}$ to $E_{2 M}^{u}$. As noted in the Introduction $p H_{k}$ is classified by

$$
Q P^{k} \stackrel{\Delta}{\longrightarrow} \cup Q P^{k_{1}} \times \cdots \times Q P^{k_{r}} \rightarrow B S p
$$

and hence it lifts to $E_{\max (2 k, M(p, k))}^{u}$.

Proof of (i) if $p=2^{i+1}$. Let $M=M(p, k)$. By hypothesis $2^{i} H_{l} \times 2^{i} H_{k-l}$ lifts to $E_{\max (2 k, M+2)}^{u}$. Assume $M \geqslant 2 k$, for otherwise we are done. As in the proof of the first case the liftings to $E_{M+3}^{u}$ are compatible, and by Theorem 2.2 there is a map $E_{M+3}^{u}{ }^{(4 k)} \rightarrow \Sigma P_{M+1}^{M+2} \wedge b u$ whose fiber has the $4 k$-type of $E_{M+1}^{u}$. Thus the lifting to $E_{M+1}^{u}$ (and hence to $E_{M}^{u}$ ) exists if and only if the top component of the composite

$$
Q P^{k} \stackrel{\Delta}{\longrightarrow} \cup Q P^{l} \times Q P^{k-l} \rightarrow E_{M+3}^{u} \rightarrow \Sigma P_{M+1}^{M+2} \wedge b u
$$

is zero, since the lower components are zero by the induction hypothesis. By Corollary 2.6(i) this is zero if and only if the top obstruction is nonzero for an even number of $2^{i} H_{l} \times 2^{i} H_{k-l}$. But the top obstruction for $2^{i} H_{l} \times 2^{i} H_{k-l}$ equals that of $2^{i} H_{k-l} \times 2^{i} H_{l}$, since the map $Q P^{k-l} \times Q P^{l} \rightarrow E_{M+3}^{u}$ can be chosen to be the composite

$$
Q P^{k-l} \times Q P^{l} \stackrel{T}{\longrightarrow} Q P^{l} \times Q P^{k-l} \stackrel{g}{\longrightarrow} E_{M+3}^{u},
$$

where $g$ is a lifting for $2^{i} H_{l} \times 2^{i} H_{k-l}$. The only unpaired obstruction is that of $2^{i} H_{k / 2} \times 2^{i} H_{k / 2}$ if $k$ is even, but this is zero by the hypothesis since $\gamma\left(\left\{\left(2^{i}, k / 2\right),\left(2^{i}, k / 2\right)\right\}\right)=0$. 
Proof of (ii). If $M\left(2^{e_{i}}, k_{i}\right) \geqslant 2 k_{i}$ for all $i$, then by hypothesis $\times 2^{e_{i}} H_{k_{i}}$ lifts to $E_{S}^{u}$, where

$$
S=\sum M\left(2^{e_{i}}, k_{i}\right) \leqslant M\left(\sum 2^{e_{i}}, \sum k_{i}\right)+2 \gamma\left(\left\{\left(2^{e_{i}}, k_{i}\right)\right\}\right)=M
$$

by Corollary 3.6.

Otherwise, choose the maximal value of $2^{e_{i}}+k_{i}$ such that $M\left(2^{e_{i}}, k_{i}\right)<2 k_{i}$. After renumbering we may assume that $i=1$. Then $X{ }^{e^{i}} H_{k_{i}}$ is classified by

$$
X Q P^{k_{i}} \stackrel{\Delta \times 1}{\longrightarrow} \bigcup_{k_{0}=0}^{k_{1}} Q P^{k_{0}} \times Q P^{k_{1}-k_{0}} \times \underset{i>2}{X} Q P^{k_{i}} \rightarrow B S p,
$$

where the second map classifies $2^{e_{1}-1} H \times 2^{e_{1}-1} H \times \mathrm{X}_{i>2} 2^{e_{i}} H$. By hypothesis $2^{e_{1}-1} H_{k_{0}} \times 2^{e_{1}-1} H_{k_{1}-k_{0}} \times \mathrm{X}_{i>2} 2^{e_{i} H_{k_{i}}}$ lifts to $E_{M}^{u}$, where

$$
\begin{aligned}
M=\max & \left(2 \sum k_{i}, M\left(\sum 2^{e_{i}}, \sum k_{i}\right)\right. \\
& \left.+2 \gamma\left(\left\{\left(2^{e_{1}-1}, k_{0}\right),\left(2^{e_{1}-1}, k_{1}-k_{0}\right),\left(2^{e_{2}}, k_{2}\right), \ldots\right\}\right)\right) \\
\leqslant \max ( & 2 \sum k_{i}, M\left(\sum 2^{e_{i}}, \sum k_{i}\right) \\
& \left.+2 \gamma\left(\left\{\left(2^{e_{1}}, k_{1}\right),\left(2^{e_{2}}, k_{2}\right), \ldots\right\}\right)+2\right)=M^{\prime} .
\end{aligned}
$$

As in the proof of (i), all obstructions (except possibly one which has zero obstruction) occur in pairs so that the lifting of $X 2^{e} i_{i} H_{k_{i}}$ from $E_{M^{\prime}+1}^{u}$ to $E_{M^{\prime}-1}^{u}$ exists.

THEOREM 3.8. (a) Let $M=M\left(\Sigma 2^{e_{i}}, \Sigma k_{i}\right)+2 \gamma\left(\left\{\left(2^{e^{i}}, k_{i}\right)\right\}\right)$. If $M>2 \Sigma k_{i}$. then the top obstruction for lifting $X 2^{e_{i}} H_{k_{i}}$ to $E_{M-1}^{u}$ is nonzero if and only if $M\left(\Sigma 2^{e_{i}}, \Sigma k_{i}\right)=\Sigma M\left(2^{e_{i}}, k_{i}\right)-2 \gamma\left(\left\{\left(2^{e_{i}}, k_{i}\right)\right\}\right)$ and for all $i, M\left(2^{e_{i}}, k_{i}\right)=4 k_{i}-$ $2 \nu\left(2^{e_{i}}, k_{i}\right)$.

(b) If $M(p, k)>2 k$, then the top obstruction for lifting $p H_{k}$ to $E_{M(p, k)-1}^{u}$ is nonzero if and only if $M(p, k)=4 k-2 v(p, k)$.

Proof. As in Theorem 3.7 it suffices to prove:

(i) If (a) is true whenever $\Sigma 2^{e_{i}} \leqslant p$, then (b) is true.

(ii) If (b) is true whenever $p \leqslant \max \left\{2^{e_{i}}\right\}$ and (a) is true for $X 2^{e^{i}} H_{k_{i}^{\prime}}$ whenever $\Sigma 2^{e_{i}^{\prime}}=\Sigma 2^{e_{i}}$ and $\max \left\{2^{e_{i}^{\prime}}+k_{i}^{\prime}: M\left(2^{e_{i}^{\prime}}, k_{i}^{\prime}\right) \leqslant 2 k_{i}^{\prime}\right\}<\max \left\{2^{e_{i}}+k_{i}\right.$ : $M\left(2^{e_{i}}, k_{i}\right) \leqslant 2 k_{i}$ \}, then (a) is true.

Proof of (i) if $p$ is not a power of 2. Write $p=\Sigma 2^{e_{i}}$ with $e_{i}$ distinct. The top obstruction for $p H_{k}$ is nonzero if and only if there is an odd number of $\left\langle k_{i}\right\rangle$ such that $\mathrm{X}_{2}{ }^{e_{i}} H_{k_{i}}$ has nonzero top obstruction (for lifting to $E_{M(p, k)-1}^{u}$ ). The top obstruction is certainly zero for those $\left\langle k_{i}\right\rangle$ for which $\Sigma M\left(2^{e_{i}}, k_{i}\right)<M\left(\Sigma 2^{e_{i}}, \Sigma k_{i}\right)$, 
so we need consider only those $\left\langle k_{i}\right\rangle$ for which $\Sigma M\left(2^{e_{i}}, k_{i}\right)=M\left(\Sigma 2^{e_{i}}, \Sigma k_{i}\right)$. Then

$$
\sum M\left(2^{e_{i}}, k_{i}\right)=M(p, k) \geqslant 4 k-2 \nu(p, k) \geqslant \sum\left(4 k_{i}-2 \nu\left(2^{e_{i}}, k_{i}\right)\right) .
$$

If $M(p, k)>4 k-2 v(p, k)$, then $\Sigma\left(M\left(2^{e i}, k_{i}\right)-\left(4 k_{i}-2 v\left(2^{e i}, k_{i}\right)\right)\right)>0$, so for some $i, M\left(2^{e_{i}}, k_{i}\right)>4 k_{i}-2 v\left(2^{e_{i}}, k_{i}\right)$ and hence by hypothesis $X 2^{e_{i}} H_{k_{i}}$ has top obstruction zero. Since this is true for all $\left\langle k_{i}\right\rangle, p H_{k}$ has top obstruction zero by Corollary $2.6(\mathrm{i})$.

If $M(p, k)=4 k-2 v(p, k)$, then $\times 2^{e_{i}} H_{k_{i}}$ has nonzero top obstruction if and only if for all $i, M\left(2^{e_{i}}, k_{i}\right)=4 k_{i}-2 v\left(2^{e_{i}}, k_{i}\right)$ if and only if $\nu(p, k)=$ $\Sigma \nu\left(2^{e_{i}}, k_{i}\right)$, and by Theorem 3.2 this happens for an odd number of $\left\langle k_{i}\right\rangle$. (Note that $\left\{\left\langle k_{i}\right\rangle: \nu(p, k)=\Sigma \nu\left(2^{e_{i}}, k_{i}\right)\right\} \subset\left\{\left\langle k_{i}\right\rangle: M(p, k)=\Sigma M\left(2^{e_{i}}, k_{i}\right)\right\}$.) Thus $X 2^{e^{i}} H_{k_{i}}$ has nonzero top obstruction.

Proof of (i) if $p=2^{i+1}$. Let $M=M(p, k)$. There is a commutative diagram

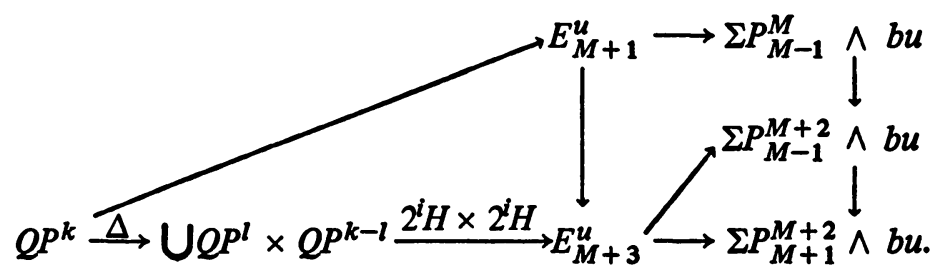

By Theorem 2.4(ii) and Corollary 2.6(i), $2^{i+1} H_{k}$ has nonzero top obstruction in $\pi_{4 k}\left(\Sigma P_{M-1}^{M} \wedge b u\right)$ if and only if it has nonzero top obstruction in $\pi_{4 k}\left(\Sigma P_{M-1}^{M+2} \wedge b u\right)$ if and only if the sum of the top obstructions for the $2^{i} H_{l} \times$ $2^{i} H_{k-l}$ in $\pi_{4 k}\left(\Sigma P_{M-1}^{M+2} \wedge b u\right)$ is nonzero. If $\left(2^{i}, l, k-l\right)$ is not special, then $2^{i} H_{l} \times 2^{i} H_{k-l}$ lifts to $E_{M}^{u}$ and hence its top obstruction in $\pi_{4 k}\left(\Sigma P_{M-1}^{M+2} \wedge b u\right)$ is divisible by 2 , so that the sum of the top obstructions for $2^{i} H_{l} \times 2^{i} H_{k-l}$ and $2^{i} H_{k-l} \times 2^{i} H_{l}$ is zero. (If $k$ is even, $2^{i} H_{k / 2} \times 2{ }^{i} H_{k / 2}$ lifts to $E_{M(p, k)-1}^{u}$.) If $\left(2^{i}, l, k-l\right)$ is special but $\{l, k-l\} \cap\left\{0,2^{i}\right\}$ is empty, then by the induction hypothesis $2^{i} H_{l} \times 2^{i} H_{k-l}$ has zero top obstruction for lifting to $E_{M+1}^{u}$, so that the sum of the obstructions in $\pi_{4 k}\left(\Sigma P_{M-1}^{M+2} \wedge b u\right)$ for $2^{i} H_{l} \times 2^{i} H_{k-l}$ and $2^{i} H_{k-l} \times$ $2^{i} H_{l}$ is zero. The only case remaining is $2^{i} H_{\epsilon 2^{i}} \times 2^{i} H_{k-\epsilon 2^{i}}$, where $\epsilon=0$ if $k \leqslant 2^{i}$ and $\epsilon=1$ if $k>2^{i}$. The sum of the obstructions in $\pi_{4 k}\left(\Sigma P_{M-1}^{M+2} \wedge b u\right)$ from this and its transpose is zero if and only if the top obstruction for lifting $2^{i} H_{\epsilon 2^{i}} \times 2^{i} H_{k-\epsilon 2^{i}}$ to $E_{M+1}^{u}$ is zero if and only if $M\left(2^{i}, k-\epsilon 2^{i}\right)>4\left(k-\epsilon 2^{i}\right)-$ $2 v\left(2^{i}, k-\epsilon 2^{i}\right)$ if and only if $M\left(2^{i+1}, k\right)>4 k-2 v\left(2^{i+1}, k\right)$.

Proof of (ii). If $M\left(2^{e^{i}}, k_{i}\right)>2 k_{i}$ for all $i$, this follows from Corollary 2.6(ii). Otherwise, the proof follows by combining the methods of Theorem 3.6(ii) and the case $p=2^{i+1}$ above.

In order to deduce Theorem 1.8, we need the following lemma, whose proof is similar to that which will be used in Theorem 1.8. 
LEMMA 3.9. For $M$ even, if a map $Q P \rightarrow B S p$ lifts to $E_{M}^{o}$ and to $E_{M-4}^{u}$, then it lifts to $E_{M-2}^{o}$.

PROoF. In the remainder of this section we shall denote $O_{k}^{n}=\Sigma P_{k}^{n} \wedge$ bo and $U_{k}^{n}=\Sigma P_{k}^{n} \wedge b u$. Suppose $M=4 m$. Consider the following commutative diagram:

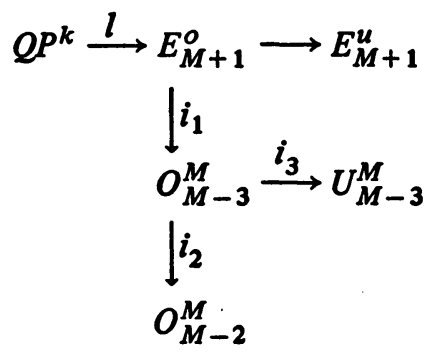

The maps of homotopy groups

$$
\pi_{4 i}\left(O_{M-2}^{M}\right) \leftarrow \pi_{4 i}\left(O_{M-3}^{M}\right) \rightarrow \pi_{4 i}\left(U_{M-3}^{M}\right)
$$

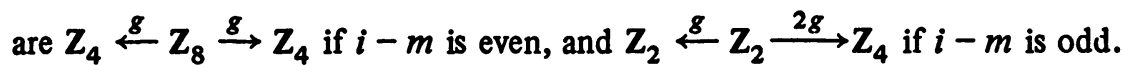
$\left[i_{3} i_{1} l\right]=0 \in \bigoplus_{i=m}^{k} \pi_{4 i}\left(U_{M-3}^{M}\right)$. Thus by Theorem 2.4(ii) $\left[i_{1} l\right]$ is divisible by 4 in $\bigoplus_{i=m}^{k} \pi_{4 i}\left(O_{M-3}^{M}\right)$ and hence $\left[i_{2} i_{1} l\right]=0$. Thus the lifting to $E_{M-2}^{o}$ exists. A similar proof works if $M=4 m+2$.

LEMMA 3.10. (i) If $M(p, k)=4 m-2$, then $N(p, k)=4 m$ if there is an $i \leqslant k$ such that $i-m$ is odd and $\nu(p, i)<v\left(\pi_{4 i}\left(\Sigma P_{4 m-3} \wedge b u\right)\right)$, and otherwise $N(p, k)=4 m-1$.

(ii) If $M(p, k)=4 m$, then $N(p, k)=4 m+2$ if there is an $i \leqslant k$ such that $i-m$ is odd and $\nu(p, i)<\nu\left(\pi_{4 i}\left(\Sigma P_{4 m-1} \wedge b u\right)\right)$, and otherwise $N(p, k)=4 m$.

Proof. If $0 \leqslant \epsilon \leqslant 3$, then

$$
\pi_{4 i}\left(\Sigma P_{4 m+\epsilon} \wedge b o\right)=2(i-m)+\left\{\begin{aligned}
1 & \text { if } \epsilon=0 \text { or } 1 \text { and } i-m \text { is odd } \\
-1 & \text { if } \epsilon=3 \text { and } i-m \text { is odd } \\
0 & \text { otherwise. }
\end{aligned}\right.
$$

Thus $\nu\left(\pi_{4 i}\left(\Sigma P_{4 m-2} \wedge b u\right)\right) \geqslant\left(\pi_{4 i}\left(\Sigma P_{4 m} \wedge b o\right)\right)$ and

$$
\begin{aligned}
\nu\left(\pi_{4 i}\left(\Sigma P_{4 m-3} \wedge b u\right)\right) & =\nu\left(\pi_{4 i}\left(\Sigma P_{4 m-2} \wedge b o\right)\right) \\
& =\nu\left(\pi_{4 i}\left(\Sigma P_{4 m-1} \wedge b o\right)\right)+ \begin{cases}1 & \text { if } i-m \text { even, } \\
0 & \text { if } i-m \text { odd. }\end{cases}
\end{aligned}
$$

(i) follows from this, and (ii) is proved similarly.

PrOOF OF THEOREM 1.8. $p H_{k}$ lifts to $\overparen{B S} p_{4 k}$ and hence to $E_{4 k}^{o}$. Thus by using Lemma 3.9, it lifts to $E_{M(p, k)+2}^{o}$. 
If $M(p, k)=4 m-2$, the obstruction for lifting $p H_{k}$ from $E_{4 m-1}^{u}$ to $E_{4 m-3}^{u}$ is an element of $\bigoplus_{i=m}^{k} \pi_{4 i}\left(\Sigma P_{4 m-3}^{4 m-2} \wedge b u\right)$. We will show below that if this class has a nonzero component for $i-m$ odd, then $p H_{k}$ does not lift to $E_{4 m-1}^{o}$, and if its $\pi_{4 i}$-component is zero for all $i$ such that $i-m$ is odd, then $p H_{k}$ lifts to $E_{4 m-1}^{o}$ but not to $E_{4 m-2}^{o}$. Combining this with Theorem 3.1 and Lemma 3.10 proves Theorem 1.8 when $M(p, k)=4 m-2$.

The claim follows from the commutative diagram:

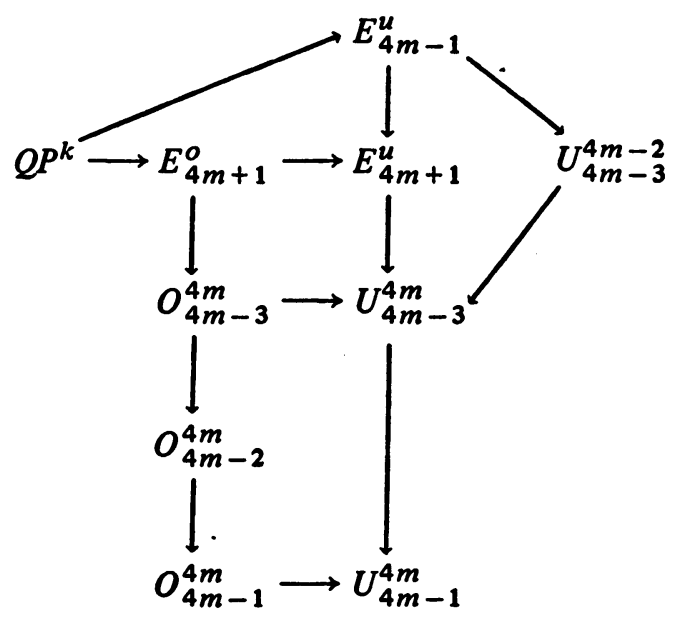

The induced maps in $\pi_{4 i}$ of the $O$ - and $U$-spaces are

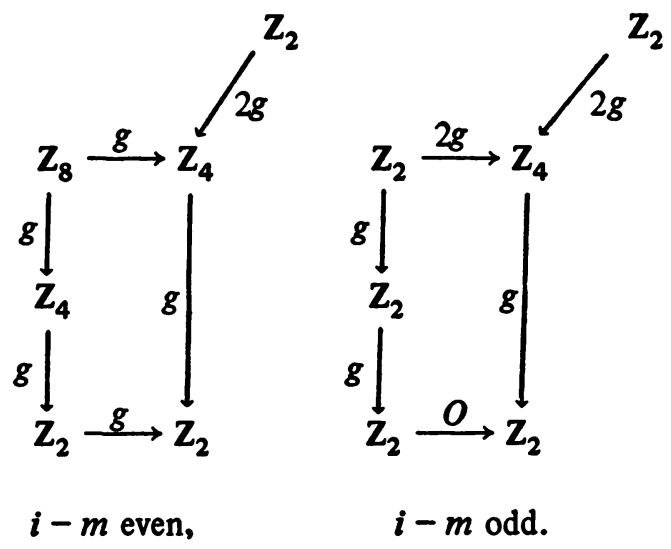

Since $p H_{k}$ lifts to $E_{4 m-1}^{u}$, the class in $\pi_{4 i}\left(U_{4 m-1}^{4 m}\right)$ is zero. Thus the class in $\pi_{4 i}\left(O_{4 m-1}^{4 m}\right)$ is zero if $i-m$ is even, while for $i-m$ odd the class in $\pi_{4 i}\left(O_{4 m-1}^{4 m}\right)$ is zero if and only if the class in $\pi_{4 i}\left(U_{4 m-3}^{4 m-2}\right)$ is zero. The claims about lifting to $E_{4 m-1}^{o}$ follow, since $p H_{k}$ lifts to $E_{4 m-1}^{o}$ if and only if the class in $\bigoplus_{4 i}\left(O_{4 m-1}^{4 m}\right)$ is zero. Since $p H_{k}$ does not lift to $E_{4 m-1}^{u}$, the class in $\pi_{4 i}\left(U_{4 m-3}^{4 m-2}\right)$ is nonzero for some $i$, and for either parity this shows the class in $\pi_{4 i}\left(O_{4 m-2}^{4 m}\right)$ is nonzero. Hence $p H_{k}$ does not lift to $E_{4 m-2}^{o}$. 
If $M(p, k)=4 m$, the theorem is proved similarly by showing that $p H_{k}$ lifts exactly to $E_{4 m+2}^{o}$ if the obstruction in $\pi_{4 i}\left(U_{4 m-1}^{4 m}\right)$ is nonzero for some $i$ such that $i-m$ is odd, and otherwise it lifts exactly to $E_{4 m}^{o}$. This follows as before from the diagram

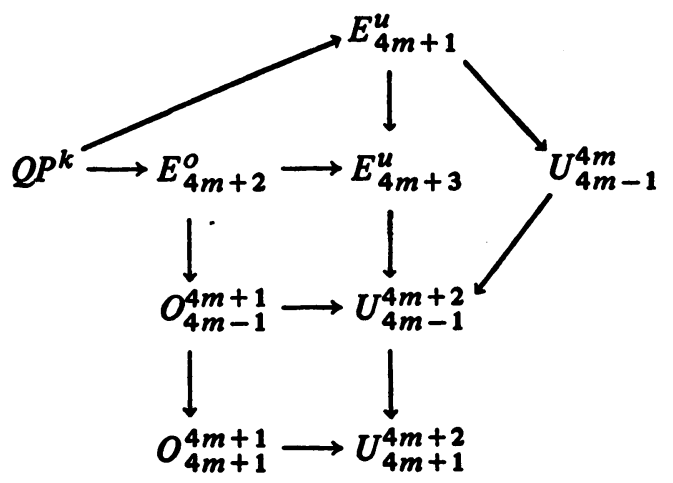

where the homomorphisms of $\pi_{4 i}$ are

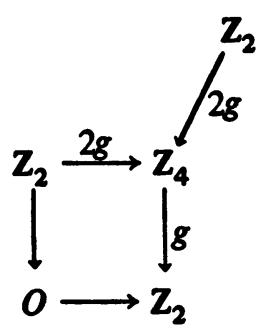

$i-m$ even,

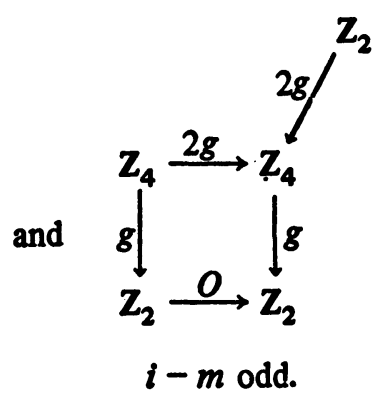

4. Proof of Theorems 1.3(b) 1.4, and 3.2. The following proposition is easily verified.

Proposition 4.1. (i) $v(m, n)=\alpha(n)+\alpha(m-n)-\alpha(m)$;

(ii) $\alpha\left(2^{i}-n\right)=i-\alpha(n-1)$;

(iii) $\alpha(n-\Delta)=\alpha(n)-\Delta+\sum_{j=0}^{\Delta-1} v(n-j)$.

THEOREM 4.2. If $p<2^{l}, 0 \leqslant l \leqslant 2^{l}$, and $0 \leqslant k-l<p$, then $v\left(2^{i}, l\right)+$ $v(p, k-l)>v\left(2^{i}+p, k\right)$. For fixed $i, p$, and $k$, there is an odd number of $l$ for which equality is achieved.

Proof (M. G. Barratr). By using Proposition 4.1(i) it is immediately verified that

$$
\begin{aligned}
\nu\left(2^{i}, l\right)+\nu(p, k-l) & =\nu\left(2^{i}+p, k\right)+\nu(k, l)+\nu\left(2^{i}+p-k, 2^{i}-l\right) \\
& >v\left(2^{i}+p, k\right) .
\end{aligned}
$$

By comparing coefficients of $x^{k}$ in $(1+x)^{2^{l}}(1+x)^{p}=(1+x)^{2^{i}+p}$, we see that 


$$
\sum_{l}\left(\begin{array}{c}
2^{i} \\
l
\end{array}\right)\left(\begin{array}{c}
p \\
k-l
\end{array}\right)=\left(\begin{array}{c}
2^{i}+p \\
k
\end{array}\right)
$$

and hence

$$
\nu\left(\sum_{l}\left(\begin{array}{c}
2^{i} \\
l
\end{array}\right)\left(\begin{array}{c}
p \\
k-l
\end{array}\right)\right)=\nu\left(2^{i}+p, k\right)
$$

The result then follows from the observation that $\nu\left(\Sigma A_{l}\right) \geqslant \min \left(\nu\left(A_{l}\right)\right)$ with equality if and only if the minimum is attained for an odd number of $l$.

Theorem 3.2 follows immediately from Theorem 4.2 by induction on the number of $\boldsymbol{e}_{\boldsymbol{i}}$.

Proposition 4.3 [15]. Let $\xi_{k}$ denote the Hopf bundle over $\mathbf{R P}^{k}$. Then

(i) $\operatorname{gd}\left(4 p \xi_{4 k+3}\right) \leqslant \operatorname{gd}\left(m H_{k}\right)$;

(ii) $\mathbf{R} P^{k} \subseteq \mathrm{R}^{k+l}$ if and only if $\operatorname{gd}\left(\left(2^{I}-k-1\right) \xi_{k}\right) \leqslant l$ for large $I$.

Proof of Theorem 1.4. Let $K=8 k+7$. Then it suffices to show $\operatorname{gd}\left(\left(2^{I}-2 k-2\right) H_{2 k+1}\right) \leqslant 8 k+7-D . v\left(\pi_{4 i-1}\left(P_{n} \wedge b o\right)\right)$ is easily seen to be $[3,7]$ given by the table

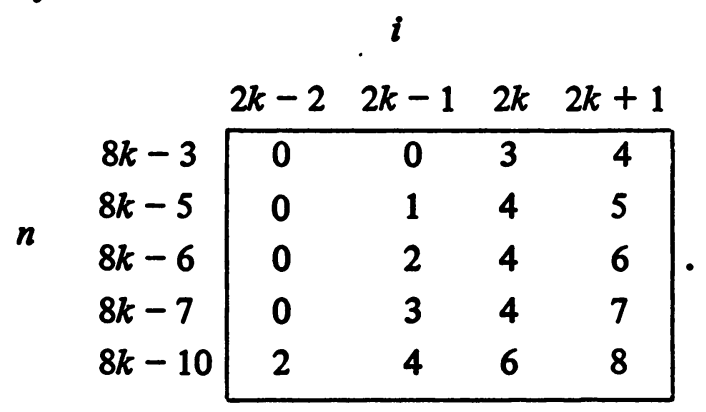

Proposition 4.1 easily implies

$$
\nu\left(2^{I}-2 k-2, i\right)= \begin{cases}\alpha(k)-1 & \text { if } i=2 k-2 \\ \alpha(k)+\nu(k)+1 & \text { if } i=2 k-1 \\ \alpha(k) & \text { if } i=2 k \\ \alpha(k)+1 & \text { if } i=2 k+1\end{cases}
$$

One can now verify that

$$
\nu\left(2^{I}-2 k-2, i\right) \geqslant \nu\left(\pi_{4 i-1}\left(P_{8 k+7-D} \wedge b o\right)\right) \text { for all } i \leqslant 2 k+1 .
$$

The condition of Theorem 1.3(b) is easily verified. A similar argument can be made when $K=8 k+3$.

ProOf OF TheOREM 1.3(b). By observing the tables of [11] and [7], we see that the condition of Theorem 1.1(b) is satisfied for $m \geqslant k-\left\{\left\{_{4}^{3}\right.\right.$ unless $m$ 
even, $\epsilon=1$ or $m$ odd, $\epsilon=2$ or 3 . In these cases we note there is a class $h_{2}^{2}$ at height 2 in dimension

$$
n+\left\{\begin{array} { l } 
{ 6 } \\
{ 9 } \\
{ 8 }
\end{array} \quad \text { if } n \equiv \left\{\begin{array}{l}
1(8) \\
6(8) \\
7(8)
\end{array}\right.\right.
$$

which is not bo-primary. (Here we use $n$ to correspond to $k$ in [11] and to our $4 m+\epsilon$.) This class is not present in the homotopy of $P_{n-\delta}$, where

$$
\delta=\left\{\begin{array} { l } 
{ 4 } \\
{ 1 } \\
{ 2 }
\end{array} \quad \text { if } n \equiv \left\{\begin{array}{l}
1 \\
6 \\
7
\end{array}\right.\right.
$$

We consider the maps of modified Postnikov towers [6] induced by the maps of fibrations

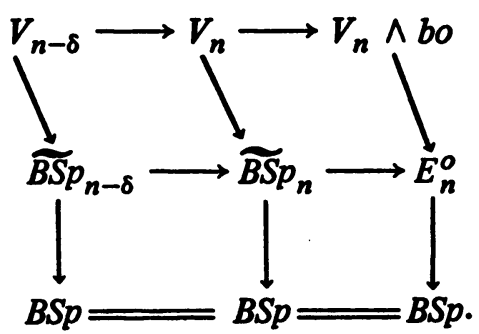

Let $E_{i}, E_{i}^{\prime}, E_{i}^{\prime \prime}$ indicate the spaces obtained in modified Postnikov towers through the dimension $4 k$ of $Q P^{k}$; this will be $\leqslant$

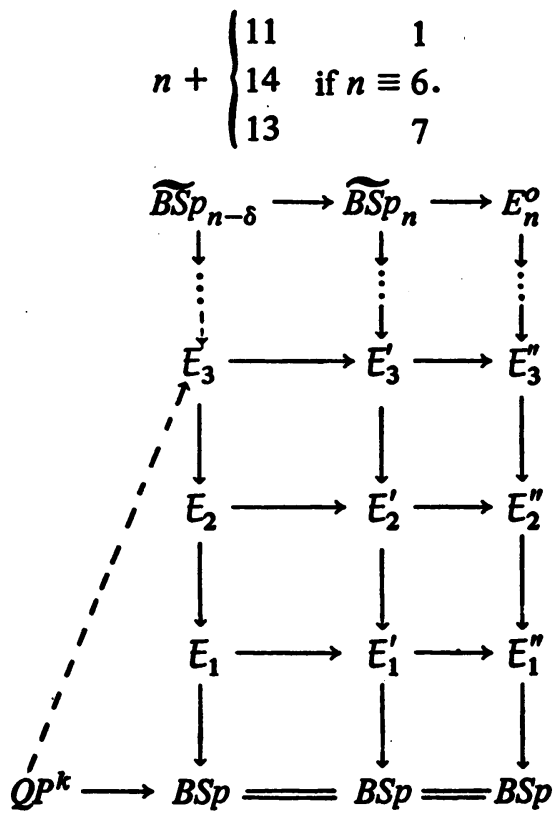


By the assumptions on $v(p, i), Q P^{k}$ lifts to $E_{3}$ and to $E_{n}^{o}$. But all $k$-invariants of $E_{i}^{\prime}, i \geqslant 3$, come from those of $E_{i}^{\prime \prime}$ and hence map to zero in $Q P^{k}$. Thus $Q P^{k}$ lifts to $\widetilde{B S} p_{n}$.

\section{REFERENCES}

1. J. F. Adams, Quillen's work on formal groups and complex cobordism, mimeographed lecture notes, Chicago, 1970.

2. D. W. Anderson, The real $K$-theory of classifying spaces, Proc. Nat. Acad. Sci. U.S.A. 51 (1964), 634-636.

3. D. M. Davis, Generalized homology and the generalized vector field problem, Quart. J. Math. Oxford Ser. (2) 25 (1974), 169-193.

4. S. Gitler, Immersion and embedding of manifolds, Proc. Sympos. Pure Math., vol. 22, Amer. Math. Soc., Providence, R. 1., 1971, pp. 87-96. MR 47 \#4275.

5. S. Gitler and M. E. Mahowald, Obstruction theory and $K$-theory (mimeograph).

6. - The geometric dimension of real stable vector bundles, Bol. Soc. Mat. Mexicana (2) 11 (1966), 85-107. MR 37 \#6922.

7. S. Gitler, M. E. Mahowald and R. J. Milgram, The nonimmersion problem for $\mathrm{R}^{\boldsymbol{n}} \boldsymbol{n}$ and higher-order cohomology operations, Proc. Nat. Acad. Sci. U. S. A. 60 (1968), $432-437$. MR 37 \#3581.

8. D. H. Husemoller, Fibre bundles, McGraw-Hill, New York, 1966. MR 37 \#4821.

9. I. M. James, Spaces associated with Stiefel manifolds, Proc. London Math. Soc. (3) 9 (1959), 115-140. MR 21 \#1596.

10. L. L. Larmore, Twisted cohomology theories and the single obstruction to lifting, Pacific J. Math. 41 (1972), 755-769.

11. M. E. Mahowald, The metastable homotopy of $S^{n}$, Mem. Amer. Math. Soc. No. 72 (1967). MR 38 \#5216.

12. R. J. Milgram, Immersing projective spaces, Ann. of Math. (2) 85 (1967), 473-482. MR 35 \#2293.

13. J. C. Moore, Some applications of homology theory to homotopy problems, Ann. of Math. (2) 58 (1953), 325-350. MR 15, 549.

14. D. Randall, Note on the generalized vector field problem, Bol. Soc. Mat. Mexicana (2) 17 (1972), 40-41.

15. B. J. Sanderson, Immersions and embeddings of projective spaces, Proc. London Math. Soc. (3) 14 (1964), 137-153. MR 29 \#2814.

16. E. H. Spanier, Function spaces and duality, Ann. of Math. (2) 70 (1959), 338-378. MR 21 \#6584.

17. G. W. Whitehead, Generalized homology theories, Trans. Amer. Math. Soc. 102 (1962), 227-283. MR 25 \#573.

DEPARTMENT OF MATHEMATICS, NORTHWESTERN UNIVERSITY, EVANSTON, ILLINOIS 60201 (Current address of M. E. Mahowald)

Current address (D. M. Davis): Department of Mathematics, Lehigh University, Bethlehem, Pennsylvania 18015 